$\underline{\xi}=$ 离

\title{
The unfortunate miss: a saddle pulmonary embolism
}

\author{
William Li *, Marek Gruca, Bhaskara Madhira \\ SUNY Upstate Medical University \\ *Corresponding author E-mail: liwi@ upstate.edu
}

\begin{abstract}
Pulmonary embolism is a potentially life-threatening condition that requires prompt diagnosis and efficient management such as in the form of thrombolysis or surgical thrombectomy. Saddle pulmonary emboli occurring at the bifurcation of the pulmonary artery are especially dangerous as they put afflicted individuals at risk for sudden hemodynamic collapse. While CT Angiography at present is the current imaging modality of choice, times exist when they are contraindicated and V/Q scintigraphy is used as the choice alternative. We present a rare catastrophic case of a saddle pulmonary embolism in an individual with a low-probabilityinterpretation on V/Q scintigraphy despite a clear depiction of the thrombus on echocardiography.
\end{abstract}

Keywords: Pulmonary; Embolus; Thrombus; Thrombolysis; Echocardiogram.

\section{Releases}

This article has never been published previously, and it is not under consideration from publication elsewhere. The publication is approved by all authors and tacitly by the responsible authorities where the work was carried out, and if accepted, it will not be published elsewhere including electronically in the same form, in English or in any other language, without the written consent of the copyright-holder.

\section{Case}

Our patient was a 70-year-oldfemale with a history of COPD, paroxysmal atrial fibrillation, congestive heart failure (HFrEF), type 2 diabetes mellitus, and non-small cell lung carcinoma (NSLCL) who presented with symptoms of bilateral lower extremity swelling, dyspnea on exertion and generalized weakness. Admitted for acutely decompensated heart failure, there was some suspicion for pulmonary embolism (PE) as the patient experienced oxygen saturation of $90 \%$ despite supplemental oxygen.Her other vital signs were otherwise unremarkable save for a heart rate of 121 beats/minute. Pertinent physical examination findings included elevated jugular venous pressure at $5 \mathrm{~cm}$ above the sternal angle, bibasilar pulmonary fine crackles, and bilateral lower extremity $1+$ pitting edema. Because of an allergy to contrast dye and low Well's score, a V/Q scan was opted for in lieu of thoracic computed tomography angiography (CTA) with results suggesting low probability of a $\mathrm{PE}$ as there were matching perfusion and ventilation defects (Figure 1). A subsequent echocardiogram performed showed extensive right ventricular (RV) dilatation with interventricularseptal bowing and an echogenic focus in the pulmonary artery (PA) concerning for a saddle PE (Figures 2 and 3). However, despite intravenous heparin infusion, the patient decompensated with acute hypoxemic respiratory failure and died after comfort care measures were agreed upon.

\section{Discussion}

Saddle PE has traditionally been known as one of the most severe forms of such and previously were more oftentimes diagnosed during autopsy because of delayed diagnosis and the short time frame in which it can cause catastrophic consequences (Pruszcyk et al. 2003). At present, CTA is the consensus initial imaging modality of choice for suspected pulmonary emboli (Bettman et al. 2012). However, V/Q scintigraphy traditionally is the choice alternative when patients have a contraindication to contrast dye. Sostman et al. noted $85 \%$ sensitivity and $73 \%$ specificity of V/Q scintigraphy amongst individuals in the group, including low probability of PE on clinical assessment(Sostman et al.2008). However, to our knowledge, the statistical measures are not clear in regards to massive PE as exhibited in our patient. One explanation for delayed diagnosis can be explained by the fact that many patients are too unstable hemodynamically to undergo CTA (Kwak et al. 2013).

One prospective observational study revealed $50 \%$ sensitivity but $98 \%$ specificity for pulmonary embolism with the presence of right ventricular dilatation (McConnell's sign) on bedside echocardiography in patients with moderate to high pretest probability. Despite having a low Well's core, echocardiography effectively visualized the suggestive finding and is a useful tool for diagnosis of PE (Dresden et al.2014). This contrasts with the PIOPED study stating a $4 \%$ probability of pulmonary embolism in individuals with low clinical probability in addition to low probability on V/Q scintigraphy (Abdelaziz et al. 2006).

At present, the mainstay of management remains anticoagulation, thrombolysis, catheter embolectomy and surgical embolectomy with the surgical option being reserved the hemodynamically unstable and those with contraindications to thrombolysis (Dauphine \&Omari 2005). Early diagnosis is paramount for management of massive pulmonary embolism as mortality rates can rise up to $70 \%$ within the first hour of presenting with coinciding degree of right ventricular dysfunction, cardiac arrest and resulting in congestive heart failure (Samoukovic et al. 2010). Our patient did not receive therapeutic treatment with anticoagulation until approxi- 
mately 3 hours after her initial presentation which arguably played a critical factor in her ultimate death. Early diagnosis with echocardiography would indeed have expedited her diagnosis prompting early therapeutic measures.

We highlight the rare case of a saddle PE clearly apparent on echocardiography though noted to have low probability on V/Q scintigraphy. In the future, we should consider the use of echocardiography as an effective modality for investigation of pulmonary emboli in those with clinical suspicion.

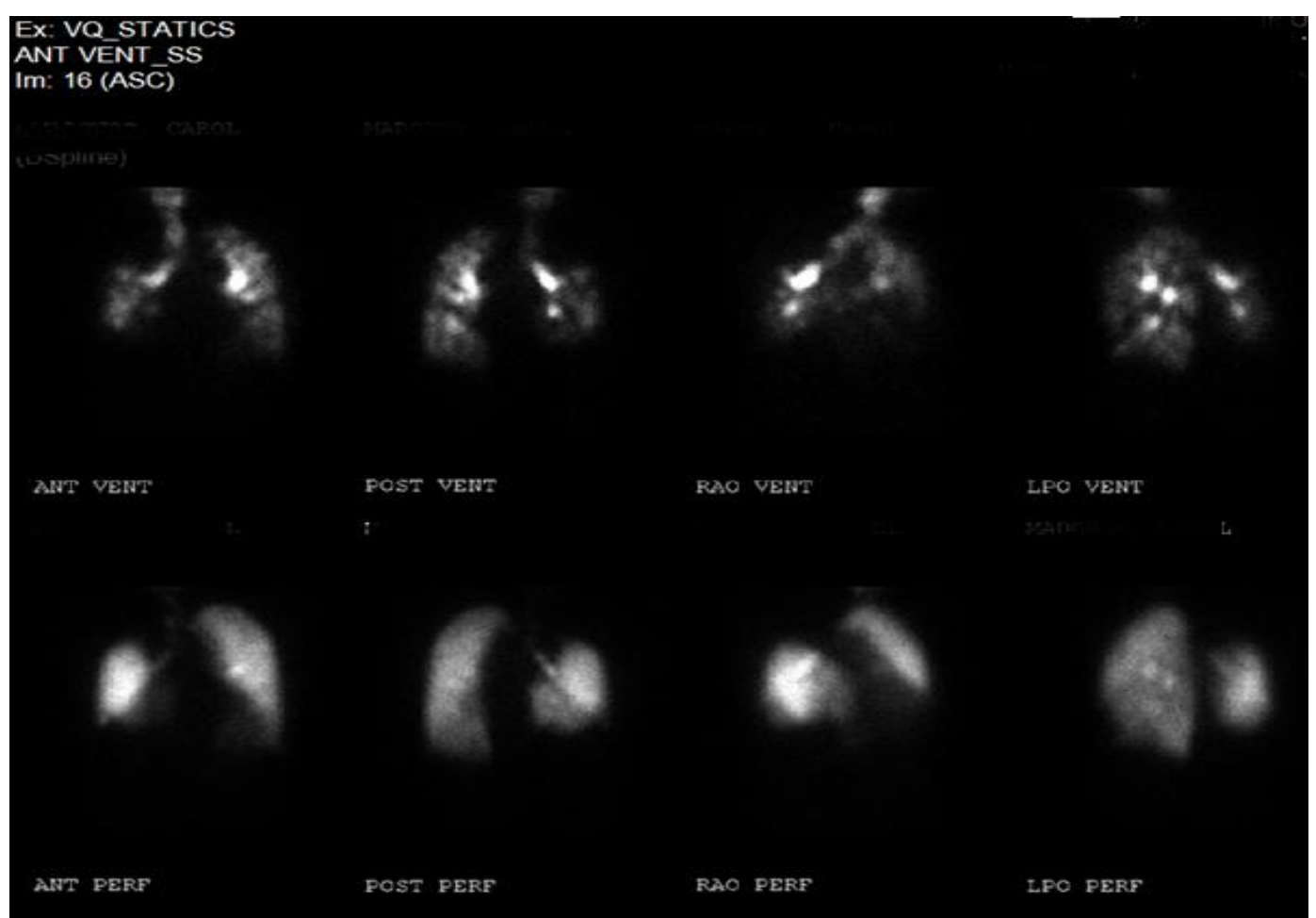

Fig. 1:V/Q ScintigraphyDemonstrating Matching Perfusion and Ventilation Defects.

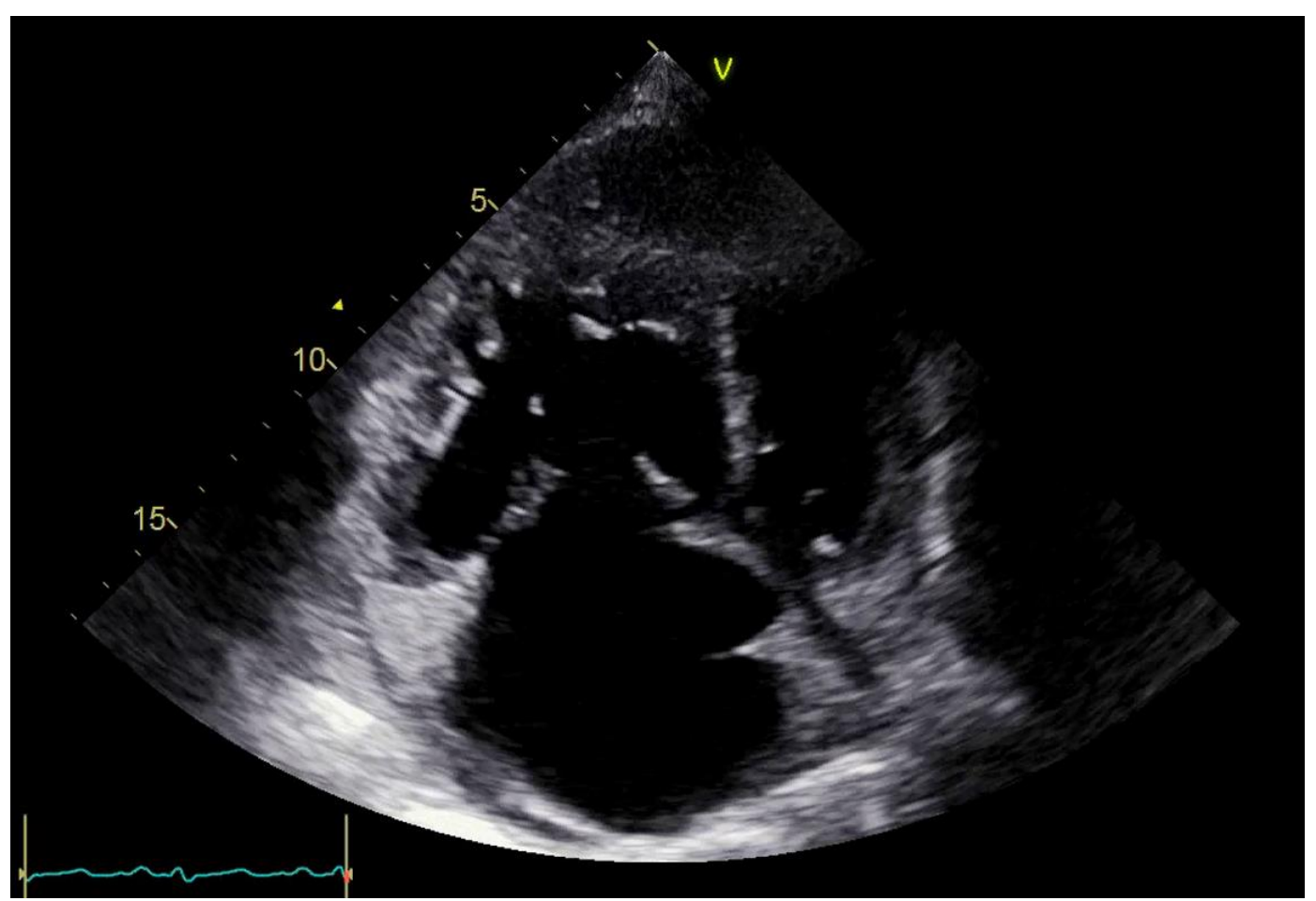

Fig. 2:Echocardiography with Extensive Right Ventricular Dilatation and InterventricularSeptal Bowing. 


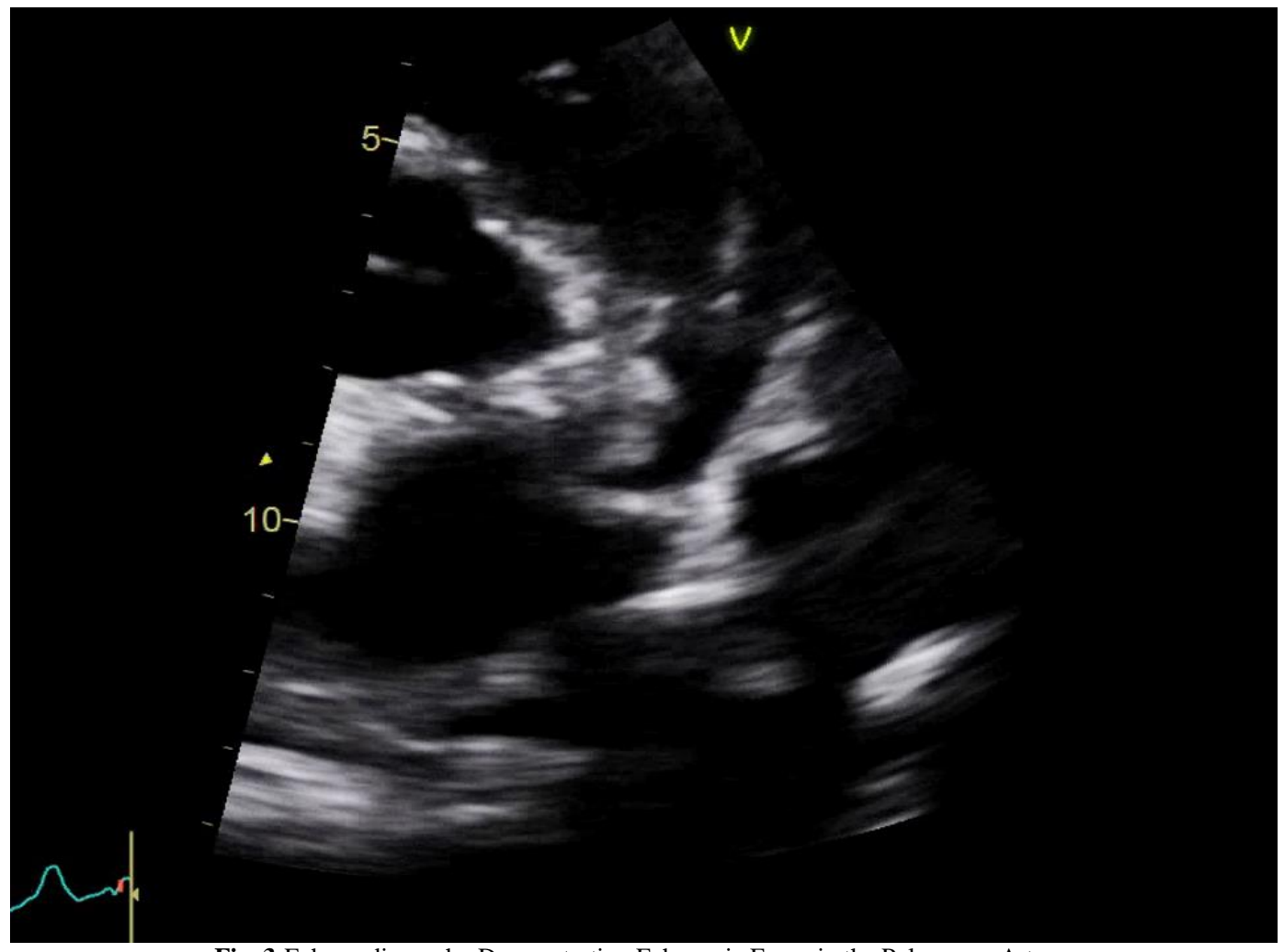

Fig. 3:Echocardiography Demonstrating Echogenic Focus in the Pulmonary Artery.

\section{Conclusion}

Saddle pulmonary embolism is a medical emergency manifesting as a large clot with the potential for causing sudden hemodynamic collapse, and as many as $25 \%$ present as sudden death out of more than 100,000 cases reported annually (Nama et al. 2016). Though CTA exists as the gold standard for diagnosis of pulmonary emboli, patients may have contraindications to such a modality. In such instances, our case highlights the value of echocardiography over V/Q scintigraphy as the modality of choice in a patient with ample clinical suspicion and who have a contraindication to CTA as V/Q scintigraphy may place a patient at low or very low probability and miss the diagnosis despite historically being a secondlinemodality of choice.

\section{References}

[1] Pruszcyk P, Pacho R, Ciurzynski M, Kurzyna M, Burakowska B, Tomkowski W (2003) Short term clinical outcome of acute saddle pulmonary embolism. Heart 89 (3), 335-336. https://doi.org/10.1136/heart.89.3.335.

[2] Bettman, MA, Baginski, SG, White RD, Woodlard, PK, Abbara S, Atalay MK, Dorbala S, Haramati LB, HendelRc, Martin ET 3rd, Ryan T, Steiner RM (2012) ACR appropriateness criteria acute chest pain-suspected pulmonary embolism. Journal of Thoracic Imaging; 27(2), 28-31. https://doi.org/10.1097/RTI.0b013e31823efeb6.

[3] Sostman, HD, Stein, PD, Gottschalk, A, Matta F, Hull R, Goodman L (2008) Acute pulmonary embolism: sensitivity and specificity of ventilation-perfusion scintigraphy in PIOPED II study. Radiology 246(3), 941-946. https://doi.org/10.1148/radiol.2463070270.

[4] Kwak M, Kim W, Lee C, Seo DW, Sohn CH, Ahn S, Lim KS Donnino MW (2013) The impact of saddle embolism on the major adverse event rate of patients with non-high-risk pulmonary embolism. The British Journal of Radiology 86 (1032). https://doi.org/10.1259/bjr.20130273.

[5] Dresden, S, Mitchell, P, Rahimi, L, Leo M, Rubin-Smith J, Bibi S, White L, Langlois B, Sullivan A, Carmody K (2014) Right ventricular dilatation on bedside echocardiography performed by emergency physicians aids in the diagnosis of pulmonary embolism Annals of Emergency Medicine 63(1), 1624.https://doi.org/10.1016/j.annemergmed.2013.08.016.
[6] Abdelaziz M, Wali S, Hamad M, Krayem A, Samman Y (2006) Pulmonary embolism: a diagnostic approach. Annals ofThoracic Medicine1(1), 31-40.https://doi.org/10.4103/1817-1737.25869.

[7] Dauphine C, Omari B (2005) Pulmonaryembolectomy for acute massive pulmonary embolism. Annals of Thoracic Surgery 79(4), 1240-1244. https://doi.org/10.1016/j.athoracsur.2004.08.081.

[8] Samoukovic G, Melas T, Varennes B (2010) the role of pulmonary embolectomy in the treatment of acute pulmonary embolism: a literature review from 1968 to 2008. Interactive CardioVascular Thoracic Surgery 11(3), 265-270. https://doi.org/10.1510/icvts.2009.228361.

[9] Nama V, Siddiqui S, Balasubramanian R, Sarasam R, Shetty V (2016) Saddle pulmonary embolism: right ventricular strain an indicator for early surgical approach. Oxford Medical Case Reports 6, 130-134.https://doi.org/10.1093/omcr/omw045. 